\title{
Aquisição de medicamentos no setor público: o binômio qualidade - custo
}

\author{
Pharmaceutical procurement by the public sector: \\ the quality/cost relationship
}

\author{
Vera Lúcia Luiza 1,2 \\ Claudia Garcia Serpa Osorio de Castro 2,3 \\ Joaquim Moreira Nunes 4
}

\footnotetext{
1 Serviço de Farmácia, Hospital Evandro Chagas, Fundação Oswaldo Cruz. Av. Brasil 4365, Manguinhos, Rio de Janeiro, $R J$ 21045-900 Brasil.

2 Núcleo de Assistência Farmacêutica, Centro

Colaborador da Organização Mundial da Saúde em Políticas Farmacêuticas, Escola Nacional de Saúde Pública. Rua Leopoldo Bulhões 1480 , sala 406, Manguinhos, Rio de Janeiro, $R J$ 21045-900, Brasil. vera@fiocruz.br

3 Servico de Farmácia, Instituto Fernandes Figueira, Av. Rui Barbosa 716 ,

Flamengo, Rio de Janeiro, $R J$ 22250-020, Brasil. claudia@iff.fiocruz.br 4 Núcleo de Tecnologia e Logística em Saúde, Centro de Saúde Escola Germano Sinval Farias, Escola Nacional de Saúde Pública, Av. Brasil 4036, sala 902, Manguinhos, Rio de Janeiro, RJ, 21045-900, Brasil. nutec@ensp.fiocruz.br
}

\begin{abstract}
The authors discuss procurement and provision of pharmaceutical products from the perspective of supply management in the public health sector, focusing on two main aspects: quality and cost. The article analyzes issues to be considered by buyers when evaluating drug quality, especially formulation stability, bioequivalence, and the role of generics. Also discussed are factors involving costs and cost management in relation to technological innovations and consumer demands. New alternatives and suggestions are examined and presented for procurement of high-quality, cost-effective drug products.
\end{abstract}

Key words Drug Costs; Drug Quality; Drugs; Public Sector

Resumo No presente trabalho, os autores abordam, dentro do âmbito da administração de materiais, a aquisição e o aprovisionamento de medicamentos no setor público de saúde, discutindo as duas principais vertentes da questão: qualidade e custo. São relatados os fatores que devem ser considerados pelo comprador quando se avalia o quesito qualidade de medicamentos, em especial a questão dos genéricos, a bioequivalência e a estabilidade das formulações. Por outro lado, o item custo é examinado, assim como as implicações que uma demanda diferenciada e voltada para as inovações tecnológicas tem sobre ele. Por fim, discutem-se alternativas, já empregadas em algumas instituições, apresentando-se sugestões cuja finalidade implica na possibilidade de compra de produtos de qualidade aliada à contenção de custos.

Palavras-chave Custos de Medicamentos; Qualidade dos Medicamentos; Medicamentos; Setor Público 


\section{Introdução}

A administração de materiais tem-se destacado como um ponto crítico, entre outros, na gestão das unidades de saúde. O setor público, particularmente, vem se preocupando de forma crescente com a questão da eficiência, resultando em uma ampla discussão da necessidade de profissionalização das ações das atividades meio.

Reconhece-se, assim, que a oferta de produtos de qualidade, em quantidades adequadas, adquiridos por um preço razoável são aspectos-chave na viabilização econômica das unidades.

O mercado brasileiro de medicamentos apresenta particularidades que fazem desta discussão um capítulo à parte. O mercado nacional congrega uma grande quantidade de medicamentos. O número total de apresentações registradas não está precisado; estima-se que o total de apresentações disponíveis beire 15 mil (Bermudez, 1992). Assim como farto em variedade de produtos, apresenta-se também bastante lucrativo: segundo o Sindicato da Indústria de Produtos Farmacêuticos (SINDUSFARM, 1998), o faturamento em dólares americanos em 1997 teria crescido 6,7\% em relação a 1996, chegando a 10,3 bilhões de dólares, apesar de menor número de unidades vendidas. A explicação da indústria foi o aumento real de preços e a incorporação de novos produtos ao mercado.

Uma competitividade saudável entre empresas que produzem e vendem medicamentos tem tido, nos países desenvolvidos, participação expressiva na redução de custos (Mehl \& Santell, 1997), desde que possa ser regulada, do ponto de vista do comprador, particularmente no que concerne à garantia de qualidade. No âmbito do serviço público no Brasil, existe farta legislação que intenta oferecer meios para regulamentação da oferta e dos processos de aquisição (Bermúdez, 1992; Brasil, 1993b; Moreira-Lima et al., 1993). No entanto, defrontase na prática com grande carência de uniformização das exigências legais e técnicas (Wexman, 1990; Osorio-de-Castro \& Ribeiro, 1996) e, ainda, com a insuficiência de meios para sua operacionalização.

\section{Qualidade}

Na aquisição de medicamentos, é desejável que estes tenham, antes de tudo, qualidade. Existem, pelos menos, duas dimensões para a qualidade desejada. A primeira diz respeito a ques- tões hoje plenamente reconhecidas e valorizadas, graças à crescente atuação dos epidemiologistas e farmacologistas clínicos, como eficácia, efetividade e adequabilidade ao perfil nosológico do serviço de saúde ou da população a que se pretende atender. Estas exigências devem ser previamente resolvidas por um bom processo de seleção e padronização (Johnson \& Bootman, 1994), objeto de uma outra discussão, que nos escapa neste trabalho. A segunda dimensão aqui focalizada trata do estabelecimento do grau de exigência de qualidade pretendido e do que pode ser efetivamente feito durante o processo de aquisição para garanti-lo.

\section{Questões ligadas ao medicamento}

O que se espera de um medicamento (fármaco mais excipientes ou veículos) é que ele tenha, no momento do uso, preservada sua ação farmacológica e que a toxicidade da formulação mantenha-se em níveis aceitáveis, conforme o determinado pelos testes que precedem sua comercialização. Tanto os princípios ativos como os veículos ou excipientes podem contribuir para situações desejáveis ou indesejáveis na terapêutica.

Considere-se, ainda, que a estabilidade da formulação dependa tanto do fármaco per se, quanto da mistura de excipientes ou veículos utilizados, assim como da interação entre ambos face às condições às quais estão submetidos. Essas condições podem se dividir em intrínsecas e extrínsecas. As primeiras, de responsabilidade do fabricante, estão relacionadas à qualidade da matéria prima, do processo produtivo e do material de embalagem. As condições extrínsecas correspondem principalmente a fatores ambientais como luminosidade, temperatura e umidade (Defelipe, 1985).

A estabilidade física se expressa pela integridade da formulação farmacêutica. São exemplos de perda de estabilidade física o amolecimento de cápsulas gelatinosas, o esfarelamento de comprimidos, a formação de caking (formação de depósito endurecido) nas suspensões, a quebra de emulsões, etc. A perda da integridade lesa as características biofarmacêuticas, levando a um inevitável comprometimento de eventos, como a liberação e a absorção do princípio ativo.

A estabilidade química é perdida com a degradação dos componentes, o que significa sempre a formação de produtos de decomposição diferentes dos originais. Disto podem decorrer dois problemas. O primeiro é a perda do efeito farmacológico, dado que a concentração do princípio ativo se distanciará da faixa tera- 
pêutica. O outro é que, algumas vezes, o produto de decomposição tem uma toxicidade própria, vindo a comprometer o efeito benéfico desejado.

Finalmente, a perda da estabilidade microbiológica pode significar tanto a contaminação por microorganismos além nos níveis admitidos para uma determinada forma farmacêutica quanto a perda da atividade biológica de um produto.

O tempo de validade de um medicamento é, assim, o período no qual o produto conserva suas propriedades de tal forma que sua estabilidade física, química e microbiológica sejam mantidas. Por fim, admite-se que as estabilidades física, química e microbiológica de um dado medicamento configurem condições mínimas para sua adequada ação farmacológica e o mínimo de ação tóxica.

A inspeção físico-macroscópica é um método que exige pouco ou nenhum equipamento e permite uma primeira triagem dos produtos, principalmente quando se incluem aspectos da rotulagem. Apesar de se poder fazer alguma inferência sobre a qualidade do medicamento a partir de características macroscópicas ou organolépticas (controle físico macroscópico não destrutivo), não se pode esquecer que este tipo de análise não permite qualquer determinação mais apurada sobre a estabilidade ou esterilidade das formas farmacêuticas. Apesar de possibilitar a detecção somente de degradações grosseiras, é bastante útil, tendo em vista a qualidade duvidosa de muito do que é oferecido no mercado farmacêutico brasileiro. Uma degradação química que possivelmente tenha originado um derivado tóxico, uma contaminação microbiológica potencialmente letal (em caso de injetáveis) ou uma concentração de princípio ativo fora da faixa permitida, no entanto, passarão despercebidas na maioria dos casos.

Com o avanço da tecnologia, produzem-se cada vez mais fármacos com estreitas margens terapêuticas, o que exige procedimentos técnicos sofisticados, e, ainda assim, insuficientes para avaliar a qualidade e estimar sua ação farmacológica. De qualquer modo, é possível arbitrar até que ponto pretende-se exigir garantias de qualidade com a inclusão de procedimentos que privilegiem desde a inspeção macroscópica e identificação até a realização de análises para determinação de uniformidade e teor percentual, de testes físico-químicos, de ensaios de bioequivalência.

\section{Questões ligadas ao binômio \\ produto-mercado}

Um dos problemas do mercado brasileiro é a extrema heterogeneidade da qualidade do parque industrial de medicamentos. Em uma tentativa de submeter as indústrias a critérios de qualidade mais uniformes, foi implantado o Programa Nacional de Inspeção das Indústrias Farmacêuticas e Farmoquímicas (PNIIFF) a partir de meados desta década. Reconhece-se o valor da iniciativa, que congregou técnicos de vários estados, mobilizando esforços importantes da Secretaria de Vigilância Sanitária do Ministério da Saúde e das Secretarias Estaduais de Saúde. Não se dirimiu, entretanto, uma dificuldade antiga dos profissionais que atuam na ponta do sistema, qual seja, o acesso a informações sistematizadas e atualizadas que permitam a orientação segura dos processos licitatórios, principalmente na fase de habilitação dos produtores. A despeito destas dificuldades, através de laboriosas consultas ao Diário Oficial da União e de documentação exigida em processos licitatórios inovadores, sabe-se que várias indústrias foram interditadas total ou parcialmente, enquanto que outras receberam advertências no sentido de atenderem às normas adequadas de fabricação, as chamadas GMP (Good Manufacturing Practices). Não cabe aqui um relato pormenorizado do programa, porém sabe-se ser o PNIIFF, por definição, mais ligado a aspectos estruturais, que não são per se suficientes para a total garantia de qualidade.

Outro problema que há muito tempo vem carecendo de uma abordagem crítica no que tange ao mercado brasileiro é a insegurança de que produtos que contêm iguais componentes químicos, mesma formulação, processo de preparo e dose, administrados em iguais condições, e, portanto, teórica e biofarmaceuticamente equivalentes, sejam também efetivamente bioequivalentes, ou seja, que tenham equivalente ação terapêutica. Produtos bioequivalentes são aqueles que, cumpridos os prérequisitos já citados, comportam-se da mesma maneira e alcançam os mesmos resultados. Em condições de pouca variabilidade, nos países desenvolvidos, com forte tradição regulatória, essa avaliação já apresenta muitas dificuldades, seja pela indigência das metodologias empregadas, seja pela necessidade de avaliações repetidas através do tempo (Levy, 1995). Como assegurar, então, que uma variação da resposta terapêutica seja conseqüência de condições clínicas peculiares e não passível de ser atribuída à (mais que provável) bioinequivalência entre os produtos utilizados? 
Nas aquisições públicas de medicamentos no nosso país, esta questão aflige, pois, dentro do critério de menor preço, cada licitação pode produzir vencedores distintos, entre produtos e fabricantes. É mesmo possível que um tratamento com um mesmo medicamento seja efetivado com produtos de lotes diferentes ou similares de fabricantes diferentes. Desconfianças quanto a marcas, sejam nacionais, de laboratório estrangeiro ou mesmo aquela da antiga CEME (Central de Medicamentos), sempre prevaleceram entre médicos, farmacêuticos e pacientes. Muitos dos produtos assim estigmatizados, entretanto, tinham origem comum: empresas transnacionais. Os testes de bioequivalência, no Brasil, limitam-se a ensaios de dissolução. Os produtos considerados de marca não teriam, desse modo, garantia real de qualidade superior em relação aos seus similares terapêuticos. Esta argumentação, irrefutável e nevrálgica, aliada à constatação das diferenças substanciais e injustificáveis de preços entre produtos similares, suscitou o nascimento da política de medicamentos genéricos, que vem tentando implantar-se no país desde 1993 (Brasil, 1993a; Bermudez, 1995). Em vez de embasar-se no critério marca como diferencial de qualidade, a idéia central que norteia a política dos medicamentos genéricos é a equivalência terapêutica, mediante comparação com especialidade de referência, e a relação custo/efetividade (CADIME, 1997).

\section{Custos}

Segundo Nunes, é a escassez de recursos, tanto no setor público quanto no privado, nos países latino-americanos que enseja a formação de melhores e mais eficazes recursos gerenciais. A pressão faz-se por serviços qualitativamente superiores a custos menores. "Pede-se que o Estado seja mais eficaz, gastando menos, exige-se que ele preste mais e melhores serviços, o que significa fazer mais com muito menos recursos" (Nunes, 1996:83).

Os principais criadores de demandas de materiais nas instituições de saúde (clientes internos) são os profissionais da equipe multidisciplinar: médicos, enfermeiros, terapeutas, técnicos de laboratório, etc. Convivendo com a pressão gerada pelo contato direto com o paciente e suas necessidades, não toleram a escassez de materiais. Por outro lado, os profissionais responsáveis pelo suprimento dos materiais, entre eles o farmacêutico, vêem-se na obrigatoriedade de satisfazer os primeiros e ainda conter custos (OPAS, 1987).
A padronização é a forma prática e recomendada pela OMS e pelos teóricos da gestão de qualidade (WHO, 1977) como alternativa segura para uniformizar a qualidade e reduzir custos. Experiências nessa linha incluem a padronização de insumos, de medicamentos e de protocolos de tratamento.

Outro possível meio de lidar com as expectativas do grupo responsável pela demanda é antecipar-se a ela, participando ativamente das discussões sobre os rumos da unidade, a evolução do seu perfil de atendimento e o planejamento das ações de saúde desenvolvidas, de modo a permitir a proposição de alternativas mais racionais de abordagem dos problemas. Por exemplo, citam-se as tentativas de construção de novas relações contratuais entre fornecedores e compradores: contratos de adesão com registros de preços, terceirização, etc.

Diante do conjunto das despesas hospitalares, estima-se que os custos com medicamentos representem parcela mais que substancial. Diversos autores relacionam percentuais entre 5\% e 20\% (Liaropoulos, 1993; Roark, 1993; Ryan, 1993). A variação pode estar relacionada ao tipo de serviço, nível de assistência e qualidade dos processos decisórios e do sistema de informações envolvendo medicamentos. Questões como pleno conhecimento da oferta, preços e racionalização da terapêutica tornam-se básicos para a sobrevivência das instituições de saúde (Gouveia, 1993; Ryan, 1993). Como citado por Laporte et al. (1997) em relação à Espanha, a despesa com medicamentos tem apresentado, nos últimos anos, um aumento superior à inflação, o que coloca em perigo a oferta eqüitativa de atenção sanitária. Mehl \& Santell (1997), examinando os custos de medicamentos nos últimos cinco anos nos Estados Unidos, diagnosticaram um incremento nos preços, junto à entrada de novos e caros agentes terapêuticos, citando uma simultânea tendência de queda no período médio de internação hospitalar e o crescente esforço de contenção dos custos para enfrentar as projeções orçamentárias.

A exigência de fornecer o que há de mais novo no âmbito da terapêutica se contrapõe aos riscos das inovações. Os métodos de monitorização de reações adversas, também chamados de métodos de farmacovigilância, entre eles o sistema de notificação espontânea e o sistema de seguimento intensivo de pacientes internados, podem auxiliar na avaliação dos riscos associados a fármacos recém introduzidos no mercado, porém não redimem os possíveis erros de julgamento por ocasião da aquisição. A morbi-mortalidade relacionada a medicamentos custa muitos bilhões de dólares por 
ano em países como os Estados Unidos (Classen et al., 1997). O aperfeiçoamento dos sistemas que amparam a utilização de medicamentos em hospitais pode contribuir significativamente para a redução dessas intercorrências, tendo o farmacêutico a oportunidade de introduzir e avaliar intervenções que gerem economia, mantendo e mesmo incrementando a qualidade da assistência (Gitlow \& Melby, 1991; Johnson \& Bootman, 1994, 1997; Abramowitz et al., 1997; Bates et al., 1997).

\section{Convivendo com o binômio qualidade-custo no serviço público}

No Brasil há farta legislação concernente à regulação de mercado, ainda que, no entanto, nem sempre esteja em harmonia com o estágio evolutivo das instituições ou das demandas sociais. Algumas vezes apresenta-se avançada e outras, retrógrada.

A tentativa de equacionar o binômio qualidade-custo tem como contexto a Lei 8666/93 (Brasil, 1993b). Também chamada de lei do menor preço, por indicar critério básico de julgamento, no entanto permite a inserção de critérios de exigências de qualidade, a partir da submissão de especificações no texto do edital, em fases distintas. A primeira delas diz respeito à habilitação do licitante e sua comprovada aptidão para desempenho de suas atividades, seja produtor ou tão somente distribuidor, o que, em última análise, dá margem ao contratante de solicitar, no documento editalício, uma série de documentos pelos quais se espera inferir a idoneidade técnica. Como exemplo, pode-se citar licença de funcionamento expedida pela Vigilância Sanitária, comprovação de regularidade para com o Conselho de Farmácia, laudo do PNIIFF, documentação específica para caso de medicamentos importados, etc. A segunda diz respeito ao produto em si e pode ser exemplificada pelo registro do produto em Diário Oficial da União e pelos laudos de qualidade.

A interpretação pouco criativa da legislação, entretanto, não libertou os contratantes do serviço público da compra de produtos de qualidade duvidosa, restritos sempre ao critério de menor preço. Algumas instituições tomaram, então, medidas no sentido de sanar suas dificuldades e, nos últimos anos, têm procurado abordar de forma comprometida a construção de editais de compra. Três exemplos são relatados a seguir.

A preocupação em fornecer produtos de qualidade a preços compatíveis com o orçamento da instituição não é recente e tem me- recido esforços isolados dentro da Fundação Oswaldo Cruz desde 1995. Objetivou-se dar continuidade às atividades de normatização de compras de medicamentos, sob uma ótica experimental e com propósito avaliador (Drummond, 1991), através de um trabalho integrado das diversas unidades assistenciais, ambulatoriais e hospitalares da instituição. Foi planejado um processo de compra único, gerido por uma unidade produtora, tendo como bases o fluxograma de compra e o edital especialmente elaborado pela equipe envolvida. Primeiramente foi elaborado um edital completo, constando de exigências legais, técnicas e contratuais, e em conformidade com a legislação, por grupo composto por representantes de diversas unidades assistenciais. A unidade centralizadora elaborou e desenvolveu o processo licitatório, em conformidade com os itens discriminados no edital. Os documentos técnicos foram analisados na fase de habilitação dos licitantes. Os que não submeteram documentação completa foram inabilitados. No ato do recebimento do material foi verificada a conformidade às exigências quanto ao prazo de validade, laudo-número de lote, acondicionamento dos produtos. A chegada do material correspondeu à entrega dos laudos, que foram analisados. Uma vez aprovados os laudos, os produtos foram distribuídos às unidades assistenciais.

O edital incluiu aspectos da habilitação técnica dos licitantes, como citado anteriormente e de qualificação dos produtos, com a padronização dos laudos de qualidade, conforme proposto pela Organização Panamericana de Saúde/Organização Mundial da Saúde (Wexman, 1990).

Considera-se que a experiência mostrouse: a) eficaz em efetivar o suprimento de muitos itens nos serviços de farmácia das unidades assistenciais da instituição, deixando mais tempo alocado, nesses setores, para atividades de assistência farmacêutica propriamente dita; a participação dos farmacêuticos na compra se deu nos momentos em que foi necessária uma intervenção de cunho técnico; b) interessante do ponto de vista institucional, promovendo a integração de unidades assistenciais sob um objetivo comum: comprar medicamentos de qualidade a preços compatíveis aos habitualmente praticados pelo mercado atacadista e, finalmente, c) moralizadora, já que todos os concorrentes submeteram-se às mesmas regras de participação e exigências, tendo como referência a legislação sanitária vigente. $\mathrm{O}$ edital, elaborado pela equipe, trouxe incremento à qualidade dos processos licitatórios da instituição. Vários itens do edital foram adaptados 
pelo setor jurídico da instituição, de modo a condicionar toda e qualquer compra a critérios mínimos de qualidade. Municípios em outros estados brasileiros, como Fortaleza e Porto Alegre têm adotado itens do edital nas compras de medicamentos.

No Instituto Nacional do Câncer, uma iniciativa corrente e diferenciada é o fato de que, caso o produto apresente problemas durante sua utilização, o ônus da análise é imputado ao licitante. Isto parece resolver um problema da maioria dos setores assistenciais, que buscam por seus próprios meios obter os laudos, sempre demorados, de laboratórios de referência, arcando não somente com esse custo como também com o ônus de um possível desabastecimento durante o tempo de quarentena do produto.

No município do Rio de Janeiro, além da inserção de alguns requisitos técnicos nos editais, o procedimento de compra caracteriza-se pelo sistema de adesão e registro de preços. Assim, os contratos são efetivados de forma centralizada, com entrega descentralizada, nas unidades assistenciais. Nesses contratos, os fornecedores se obrigam, durante o período da vigência, a cobrar como valor máximo o correspondente a $70 \%$ do preço de cada item constante das listas do fabricante.

\section{Discussão}

No nosso país a compra de medicamentos no setor público vem agora encontrar uma pressão popular a favor de uma gestão mais comprometida e responsável (Antunes, 1998), o que alia necessariamente o quesito qualidade ao custo.

Recentemente, a Secretaria de Vigilância Sanitária pronunciou-se através de legislação específica sobre a questão da qualidade no ato da compra de medicamentos. Foram publicadas as Portarias 1.818 (Brasil, 1997), 2.814 (Brasil, 1998a) e 3.765 (Brasil, 1998b), que fazem recomendações gerais quanto às exigências mínimas em edital, conjugando-se à recente e ruidosa exposição pela mídia de casos de medicamentos falsificados à venda em estabelecimentos comerciais e comprados por hospitais (CRF-RJ, 1998; Werneck et al., 1998). Apesar do exemplo extremado que ações criminosas configuram, entende-se que, para o paciente, para o farmacêutico e para as instituições, as conseqüências oriundas da compra de medicamentos falsificados equivalem na prática às conseqüências geradas pela compra de medicamentos de baixa qualidade: riscos incalculáveis para a saúde do paciente, prejuízos financeiros às unidades e, para o farmacêutico, além de pro- blemas de ordem legal e até o possível impedimento de seu exercício profissional, o descrédito de seu ofício perante a comunidade e demais profissionais de saúde. Felizmente, tanto a compra de medicamentos falsificados quanto a de medicamentos de qualidade duvidosa estão sujeitas a mecanismos de prevenção.

O cenário dessa discussão contempla aspectos relativos ao sistema de abastecimento de organizações prestadoras de cuidados à saúde, com pontuação no mercado de medicamentos, sendo permeada pela relação entre dois segmentos eminentemente distintos. De um lado estão organizações voltadas para vender, constituídas por profissionais treinados, usualmente bem pagos por volume de vendas - os fornecedores. Já as organizações compradoras, nesse caso, organizações públicas de saúde, são empresas onde, via de regra, a missão institucional não está bem definida, as atividades meio são pouco profissionalizadas e os funcionários, conseqüentemente, mal treinados.

Os fornecedores são os detentores de um bem que se deseja adquirir, mas devem também ser percebidos como aqueles que, na realidade, precisam vender. A importância está em que não deve existir relação de submissão ao fornecedor.

O quadro é deveras desfavorável ao serviço público: de um lado fornecedores bem equipados; de outro, uma organização constituída de servidores mal treinados e freqüentemente desmotivados, atendendo não aos interesses de seus clientes/usuários, mas sim exclusivamente a interpretações da legislação. Muito possivelmente, um dos motivos que têm levado ao surgimento de alternativas administrativas, como fundações de apoio, organizações sociais, cooperativas de gestão, entre outras, é a baixa resolutividade das atividades meio. Nas organizações de saúde, pela natureza do objeto de trabalho, as contradições e o distanciamento entre o desejo do gestor e a realidade institucional são capazes de produzir malefícios incalculáveis à saúde dos clientes/usuários do sistema. Quantos pacientes deixam de ser adequadamente tratados ou diagnosticados por falta de produtos indicados para tal finalidade?

\section{Sugestões}

Finalmente, que ações podem ser encetadas no serviço público para melhorar o quadro vigente no que tange às relações com fornecedores de materiais ou mesmo dos serviços com seus sistemas de abastecimento? 


\section{Conhecer o fornecedor}

Constituir cadastro qualificado de fornecedores de medicamentos, com base na construção de um sistema de informações que aborde: capacidade jurídica, idoneidade, capacidade financeira e adequação técnica.

\section{Conhecer o produto}

Os medicamentos constituem um grupo de produtos com grande número de especificidades técnicas. É importante que no processo de logística estejam envolvidos profissionais capacitados - farmacêuticos e profissionais técnicos adequadamente treinados. As observações fundamentadas, de quem utiliza o produto nas organizações, devem ser utilizadas como balizadores adicionais da qualidades dos insumos.

\section{Estabelecer regras claras com} os fornecedores e cumpri-las

O processo de compras deve ser permeado por compromissos claramente estabelecidos entre comprador e fornecedor. Se a organização não cumpre as suas obrigações, não deve esperar ter bons fornecedores. Pagar em dia é importante.
Constituir um sistema de compras em que os compradores sejam facilmente identificados

Os processos de compras usualmente praticados pelo serviço público são muito burocratizados. É praticamente impossível imputar responsabilidade por uma compra mal feita. O comprador adequadamente selecionado e treinado deve ser o responsável pelas compras.

\section{Comentários finais}

Um grande desafio que é colocado ao setor público de saúde hoje é o suprimento adequado das necessidades de medicamentos, compreendendo-se os critérios de qualidade e preço.

Apesar das dificuldades que a heterogeneidade do parque industrial de medicamentos no Brasil apresenta, resultando em produtos de toda sorte, a legislação brasileira oferece opções que permitem ao farmacêutico interessado uma saída para o impasse. É possível efetivar a compra de medicamentos providos de qualidade a preços de mercado. É ainda possível especificar as características desejáveis no produto comprado, trazendo ao processo de compra o peso da experiência técnica do profissional farmacêutico. A este cabe empenharse ao máximo para proteger o paciente do uso de medicamentos de qualidade espúria, ao mesmo tempo em que também protege os interesses econômicos da instituição, o que, em última análise, reverterá no uso mais racional dos medicamentos. À instituição cabe apoiar e incentivar seus esforços, resguardando o paciente e os interesses do Estado.

\section{Referências}

ABRAMOWITZ, P. W.; WOLLER, T. W.; DANIELS, C. E.; SOMANI, S. M.; PHELPS, P. \& McCOMB, R. C., 1997. Multidisciplinary approach to reducing costs in a health system. American Journal of Health-System Pharmacy, 54:1196-1199.

ANTUNES, L., 1998. TCE anula edital de Caxias para compra de remédios. O Globo, Rio de Janeiro, 4 jun. Seção Rio, p.14.

BATES, D. W.; SPELL, N.; CULLEN, D. J.; BURDICK, E.; LAIRD, N.; PETERSEN, L. A.; SMALL, S. D.; SWEITZER, B. J. \& LEAPE, L. L., 1997. The costs of adverse drug events in hospitalized patients. JAMA, 277:307-311. 
BERMUDEZ, J. A. Z., 1992. Remédios: Saúde ou Indústria? A Produção de Medicamentos no Brasil. Rio de Janeiro: Editora Relume Dumará.

BERMUDEZ, J. A. Z., 1995. Indústria Farmacêutica, Estado e Sociedade. São Paulo: Editora Hucitec/ SOBRAVIME (Sociedade Brasileira de Vigilância de Medicamentos).

BRASIL, 1993a. Decreto no 793 de 05 de abril de 1993. Altera os Decretos no 74.170 , de 10 de junho de 1974, e no 79.094, de 05 de janeiro de 1977, que regulamentam, respectivamente, as Leis no 5.991 de 17 de janeiro de 1973 e no 6.360 de 23 de setembro de 1976 e dá outras providências. Brasília, D.F.: Diário Oficial da República Federativa do Brasil, no 65, seção 1, pp. 4397-4398, de 06/04/93.

BRASIL, 1993b. Lei no 8.666, de 21 de junho de 1993. Regulamenta o artigo 37, inciso XXI, da Constituição Federal, institui normas para licitações e contratos da Administração Pública e dá outras providências. Brasília, D.F.: Diário Oficial da República Federativa do Brasil, no 116, seção 1, pp. 82698281, de 20/06/93.

BRASIL, 1997. Ministério da Saúde. Portaria no 1.818, de 02 de dezembro de 1997. Brasília, D.F.: Diário Oficial da República Federativa do Brasil, no 234, seção 1, p. 28467, de 03/12/97.

BRASIL, 1998a. Ministério da Saúde. Portaria no 2.814, de 29 de maio de 1998. Brasília, D.F.: Diário Oficial da República Federativa do Brasil, no 221-E, seção 1, p. 7, de 18/11/98.

BRASIL, 1998b. Ministério da Saúde. Portaria no 3.765, de 20 de outubro de 1998. Brasília, D.F.: Diário Oficial da República Federativa do Brasil, no 202E, seção 1, p. 5, de 22/10/98.

CADIME (Centro Andaluz de Información de Medicamentos), 1997. Medicamentos genéricos: La política de los sin marca. Boletín Terapéutico Andaluz, 13:13-16.

CLASSEN, D. C.; PESTOTNIK, S. L.; EVANS, R. S.; LLOYD, J. F. \& BURKE, J. P., 1997. Adverse drug events in hospitalized patients. Excess length of stay, extra costs, and attributable mortality. Journal of the American Medical Association, 277:301306.

CRF-RJ (Conselho Regional de Farmácia do Estado do Rio de Janeiro), 1998. Fraudes de medicamentos: Crime hediondo. Folha do Farmacêutico, 28:3.

DEFELIPE, C. R., 1985. Estabilidade de Medicamentos. Condições Ambientais Adequadas para Conservação dos Medicamentos. Rio de Janeiro: Faculdade de Farmácia, Universidade Federal do Rio de Janeiro. (mimeo.)

DRUMMOND, M., 1991. Economic Evaluation of Pharmaceuticals: Science or Marketing? Discussion Paper 91. Heslington: Centre for Health Economics, Health Economics Consortium, University of York.

GITLOW, H. S. \& MELBY, M. J., 1991. Framework for continuous quality improvement in the provision of pharmaceutical care. American Journal of Hospital Pharmacy, 48:1917-1925.

GOUVEIA, W. A., 1993. Executive summary. In: The Rising Costs of Pharmaceuticals: Understanding and Managing the Escalating Costs of Pharmaceuticals in Health Care Institutions (Symposium). American Journal of Hospital Pharmacy, 50:S2-S3.
JOHNSON, J. A. \& BOOTMAN, J. L., 1994. Pharmacoeconomic analysis on formulary decisions: An international perspective. American Journal of Hospital Pharmacy, 51:2593-2598.

JOHNSON, J. A. \& BOOTMAN, J. L., 1997. Drug related morbidity and mortality and the economical impact of pharmaceutical care. American Journal of Health-System Pharmacy, 54:554-558.

LAPORTE, J. R.; CAPELLÀ, D.; ARNAU, J. M.; AGUSTI, A.; BOSCH, M.; CARNÉ, X.; DIOGENE, E.; FIGUERAS, A.; IBAÑEZ, L.; VALLANO, A. \& VIDAL, X., 1997. El gasto en medicamentos (2). Butlletí Groc, 10.

LEVY, G., 1995. The clay feet of bioequivalence testing. Journal of Pharmacy and Pharmacology, 47: 975-977.

LIAROPOULOS, L. L., 1993. Cost savings through technological change in the administration of antibiotics. International Journal of Technology Assessment in Health Care, 9:580-587.

MEHL, B. \& SANTELL, J. P., 1997. Projecting future drug expenditures - 1997. American Journal of Health-Systems Pharmacy, 54:153-161.

MOREIRA-LIMA, L. F.; MELLO, A. L.; MUSSOI, A. S.; GOMES, C.; PAZ, E. P. \& MOURA, M. L., 1993. Vigilância Sanitária de Medicamentos e Correlatos. Rio de Janeiro: Qualitymark.

NUNES, J. M., 1996. Mais e melhor com recursos escassos. In: Formação de Pessoal de Nível Médio para a Saúde: Desafios e Perspectivas (A. Amâncio Filho \& M. C. G. B. Moreira, org.), pp. 83-91, Rio de Janeiro: Editora Fiocruz.

OPAS (Organización Panamericana de la Salud), 1987. Enfoque de Sistemas en la Administración de Materiales en Hospitales, Washington, D.C.: OPAS/ OMS.

OSÓRIO-DE-CASTRO, C. G. S. \& RIBEIRO, D. C. S., 1996. Protocolo para aquisição de medicamentos em instituições públicas de saúde. Revista Brasileira de Farmácia, 77:85-87.

ROARK, M., 1993. Slowing rate of cost increases in the pharmacy. Hospitals, 67:56.

RYAN, B. A., 1993. The rising cost of pharmaceuticals: An industry observer's perspective. American Journal of Hospital Pharmacy, 50:S3-S4.

SINDUSFARM (Sindicato da Indústria de Produtos Farmacêuticos), 1998. Resultados do ano de 1997. Boletim SINDUSFARM, no 01, de 19/01/98.

WERNECK, A.; BOTTARI, E. \& FORTES, L., 1998. Saúde fecha farmácias na Zona Sul. O Globo, Rio de Janeiro, 3 jun, Seção Rio, p. 12.

WEXMAN, S., 1990. El Proceso de Adquisición de Medicamentos en el Sector Público. Bogotá: Editorial Trazo/Organización Panamericana de la Salud/ Organización Mundial de la Salud.

WHO (World Health Organization), 1977. The Selection of Essential Drugs: First Report of the WHO Expert Committee. WHO Technical Report Series 615. Geneva: WHO. 